\title{
Profile Structures of TJ-II Stellarator Plasmas
}

\author{
J. Herranz, ${ }^{1}$ I. Pastor, ${ }^{1}$ F. Castejón, ${ }^{1}$ E. de la Luna, ${ }^{1}$ I. García-Cortés, ${ }^{1}$ C. J. Barth, ${ }^{2}$ E. Ascasíbar, ${ }^{1}$ \\ J. Sánchez, ${ }^{1}$ and V. Tribaldos ${ }^{1}$ \\ ${ }^{1}$ Laboratorio Nacional de Fusión por Confinamiento Magnético, Asociación Euratom/Ciemat para Fusión, \\ Avenida Complutense 22, 28040 Madrid, Spain \\ ${ }^{2}$ Fom-Instituut voor Plasmafysica "Rijnhuizen," Association Euratom-Fom, Trilateral Euregio Cluster, P.O. Box 1207, \\ 3430 BE Nieuwegein, The Netherlands
}

(Received 2 June 2000)

\begin{abstract}
Fine structures are found in the TJ-II stellarator electron temperature and density profiles, when they are measured using a high spatial resolution Thomson scattering system. These structures consist of peaks and valleys superimposed to a smooth average. Some irregularities remain in an ensemble average of discharges with similar macroscopic parameters such as line density, central temperature, and plasma current. They are found in all the magnetic configurations explored in plasmas heated by electron cyclotron waves. Their characteristics are shown and their possible origin is discussed.
\end{abstract}

PACS numbers: 52.55.Hc

The appearance of fine-scale structures in the electron temperature and density profiles of a fusion device was first reported in the Rijnhuizen Tokamak Project [1]. In this device, structures were found to appear when high power microwaves at the electron cyclotron resonance were applied for heating $(\mathrm{ECRH})$. These structures are considered to be plasma filaments, with different confinement properties from the bulk plasma, that rotate at the plasma diamagnetic frequency and with a typical lifetime of a few ms. In this Letter, we present the first observations of high spatial resolution, fine-structured electron temperature, and density profiles in stellarator plasmas. The main differences with tokamak plasmas are that the magnetic configuration in stellarators is imposed by external coils and is only slightly modified by the plasma due to the diamagnetism, for the plasma pressures reached by ECRH, and the net plasma current is very small. The appearance of fine structures in both kinds of machines suggests that there could be common physical mechanisms to generate them.

The high-resolution multiposition Thomson scattering (TS) system has been used in the TJ-II stellarator $(R=$ $1.5 \mathrm{~m}, a<0.22 \mathrm{~m}, B_{0}<1.0 \mathrm{~T}, P_{\mathrm{EC}} \leq 2 \times 300 \mathrm{~kW}$, $f=53.2 \mathrm{GHz}$, and $\Delta t<300 \mathrm{~ms}$ ) [2] to measure routinely electron temperature and density profiles. The system is able to measure electron temperatures in the range of 50 to $4 \times 10^{3} \mathrm{eV}$ at 160 spatial positions separated by $2.25 \mathrm{~mm}$, each position with 90 spectral channels, along a $360 \mathrm{~mm}$ plasma chord. With this spatial resolution the system [3] is among the few able to resolve small-scale structures in the density and temperature profiles. The spectrometer and laser branch are mounted on a $9 \mathrm{~m}$ height structure, which allows a radial movement of the laser chord, making it possible for it to pass through the magnetic axis of the different TJ-II magnetic configurations. Presently, only a single profile per shot can be obtained, due to the limitation in the laser energy of $10 \mathrm{~J}$ per pulse.

In all the explored TJ-II magnetic configurations and plasma conditions, the electron temperature and density profiles measured by TS show fine spatial structures. In Fig. 1, typical temperature and density profiles are plotted for shot No. 2043, showing a peaked temperature profile and a hollow density one. The spatial width of the structures is well above the spatial resolution of the diagnostic: a single structure is mapped normally by 6 to 12 experimental points. Their amplitude can be pretty high, reaching more than $20 \%$ of the temperature value and more than $10 \%$ of the density, with respect to the smoothed profiles. An overview of the characteristics of the observed temperature structures can be seen in Table I.

To ascertain that these structures are not an experimental artifact, a detailed error source analysis of the results is performed. The main noise contribution in this TS is generated at the first image intensifier, where the conversion of a single photon coming from the plasma, into a
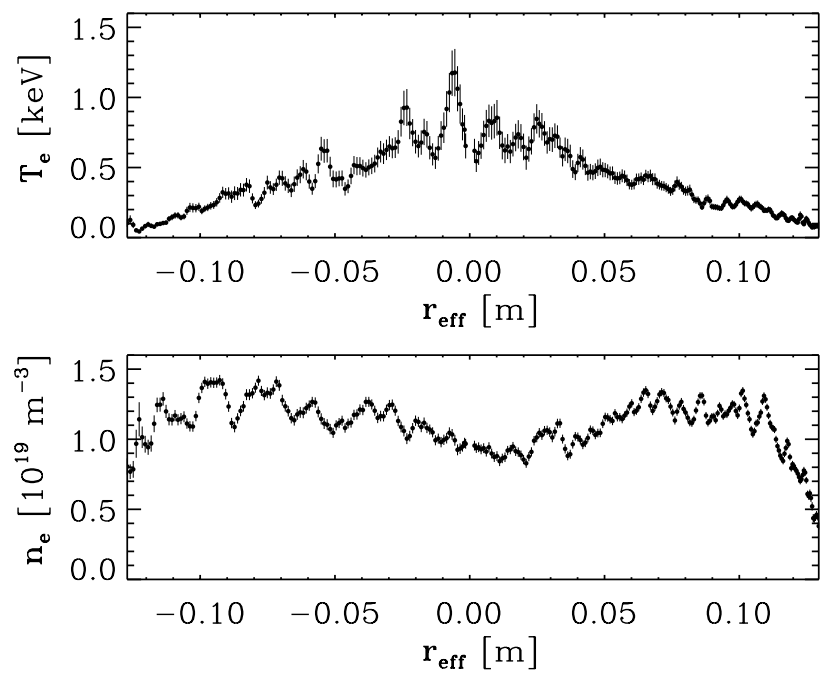

FIG. 1. Typical electron temperature (top panel) and density (bottom panel) profiles measured by the TS system in the TJ-II stellarator, showing the characteristic structures for shot No. 2043. 
TABLE I. Common temperature gradients and full width at half maximum (FHWM) of structures in some TJ-II discharges.

\begin{tabular}{ccc}
\hline \hline TJ-II No. & FWHM $(\mathrm{mm})$ & $\mathrm{eV} / \mathrm{mm}$ \\
\hline 1794 & 5 & 77 \\
1998 & 4 & 145 \\
2001 & 3 & 72 \\
2032 & 6 & 51 \\
2043 & 6 & 107 \\
2172 & 5 & 124 \\
\hline \hline
\end{tabular}

photoelectron at the photocathode of a GaAsP image tube, is assumed to follow a Poisson distribution [4,5]. Thus, the statistical spread of a spectral channel signal is determined by using the Poisson-distributed noise, with mean and variance given by $\langle n\rangle=N_{\mathrm{pe}}$, and $\sigma^{2}=N_{\mathrm{pe}}$, where $N_{\text {pe }}$ is the number of photoelectrons.

Taking into account this process, a simulation for every plasma scattering volume is performed. For all 90 channels of a single recorded spectrum, a random Poisson distribution is used to generate 200 new numerical spectra. From these spectra, 200 new temperatures are obtained. The spread in the simulated temperatures is similar to that of the $T$ error determined from the fit routine. Consequently, the statistical error cannot account for the observed structures, as can be observed in Fig. 1, where the structures are much larger than the error bars.

Another check is performed by binning sets of three pixels of the charge-coupled device (CCD) area in the spatial direction and three pixels in the wavelength direction, in order to improve the photon statistics. The result is again that the structures remain and are larger than the error bars of the electron temperature. Once the physical origin of the structures is stated, their basic properties are summarized below.

We considered a series of 15 reproducible TJ-II discharges, in which line average densities $\left(n_{l} \approx 0.95 \times\right.$ $10^{19} \mathrm{~m}^{-3}$ ), electron cyclotron emission (ECE) measured central temperatures $(T \approx 0.8 \mathrm{keV})$, and net plasma currents $(I p \approx-0.72 \mathrm{kA})$ are approximately kept constant in all the shots. The raw spectra were first averaged and afterwards fitted in this series. As can be observed in Fig. 2, the result is that temperature and density profiles are strongly smoothed by the average. However, it stands out that the structures in the profiles do not fully disappear with the average. It can be observed in the averaged temperature profile that some flattenings and small hills remain. The features of the averaged temperature profile are approximately symmetric with respect to the magnetic axis, which give some more confidence on their physical relevance. Therefore the sharp structures that appear in a single realization of the profiles can be interpreted as strong pressure oscillations superimposed over a smoother but still irregular profile.

$\Delta T(r), \Delta n(r)$, and $\Delta p(r)$, the fluctuations of the electron temperature, density, and pressure profiles, are
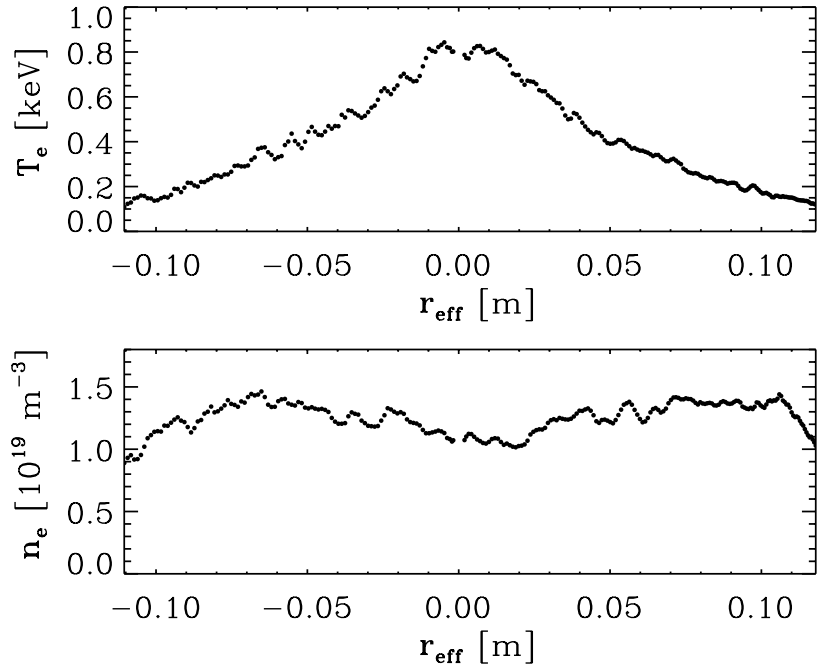

FIG. 2. Electron temperature (top panel) and density (bottom panel) profiles after averaging in a series of 15 reproducible discharges.

calculated as the experimental profiles minus the ensemble average ones. The root mean squared values of the fluctuations of temperature, density, and pressure, related to the ensemble average ones, $\operatorname{rms}(T(r)), \operatorname{rms}(n(r))$, and $\operatorname{rms}(p(r))$, are also calculated. It is observed that $\operatorname{rms}(T(r))$ is much higher at the plasma core, as is shown in Fig. 3. The $\operatorname{rms}(n(r))$ is similar along the plasma chord with almost flat density. Hence, the temperature fluctuations, as well as pressure fluctuations, are more
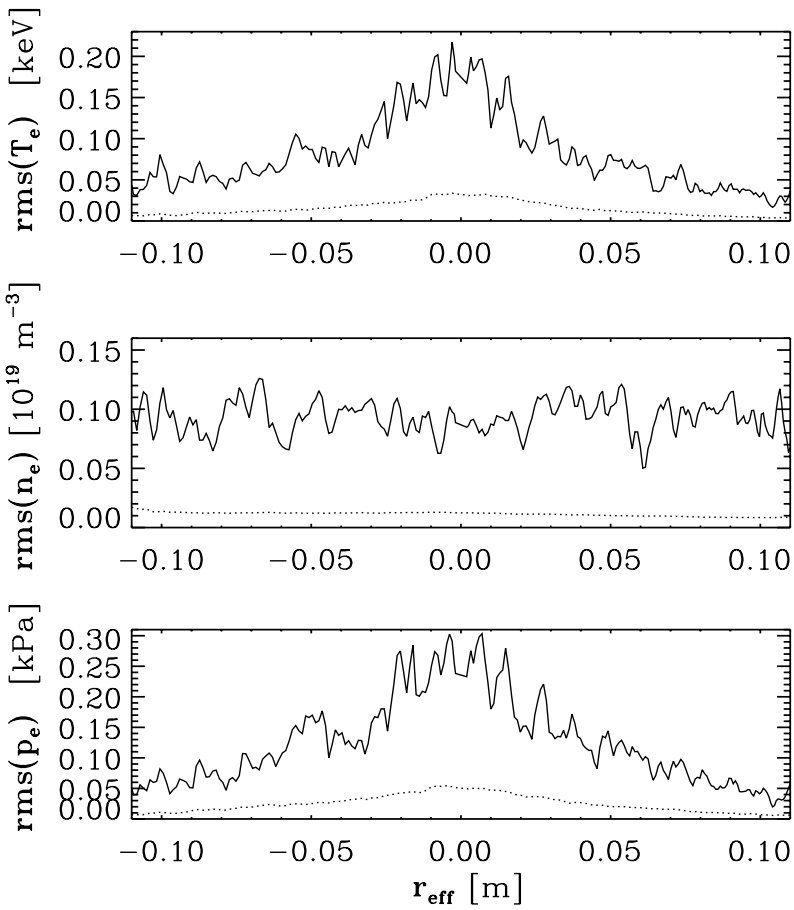

FIG. 3. $\operatorname{rms}(T(r)), \operatorname{rms}(n(r))$, and $\operatorname{rms}(p(r))$ (top, middle, and bottom panels, continuous lines) estimated for the series of 15 reproducible discharges. The dotted lines are the respective standard deviations of the $T, n$, and $p$ ensemble average ones. 


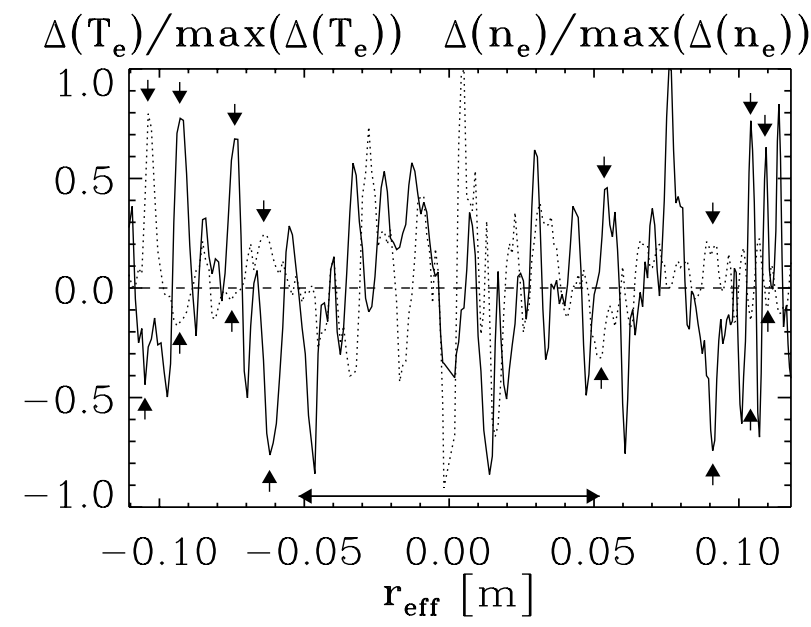

FIG. 4. Temperature, $\Delta T(r)$ (dashed line), and density $\Delta n(r)$ (continuous line), fluctuation profiles for the shot No. 3231, and the power deposition region (horizontal arrow). The vertical arrows point to the most remarkable $\Delta T(r)$ and $\Delta n(r)$ in the counterphase.

important in the plasma center, where the microwaves are absorbed. Nevertheless, the normalized quantities $\operatorname{rms}(T(r)) / T(r), \operatorname{rms}(n(r)) / n(r)$, and $\operatorname{rms}(p(r)) / p(r)$ are almost flat along the profile and take the values of $15 \%$, $10 \%$, and $18 \%$, respectively.

In most of the studied profiles $\Delta T(r)$ and $\Delta n(r)$ seem to be roughly in a counterphase, outside the power deposition profile, as can be observed in Fig. 4 for shot No. 3231. This suggests that the behavior of the $T$ and $n$ structures is different inside and outside the power deposition profile.

Every single profile can be fitted to the function $T(r)=$ $T_{0} \exp \left(-(r / a)^{2}\right)\left[1+A(r / a)^{2}+B(r / a)^{4}\right]$, where $a$ is the effective minor radius and $T_{0}, A$, and $B$ must be estimated in the fitting. $\Delta T(r)$ can then be introduced as the difference between the real profile and the fitting. A global $\operatorname{rms}(T)$ has been calculated for each profile to have a characterization of the fluctuation magnitude in a single profile. With this purpose, $\operatorname{rms}(T)$ has been calculated for 54 different plasma profiles, showing a high correlation with the electron collisionality: $\operatorname{rms}(T) \alpha 1 /\left(n / T^{3 / 2}\right)$, with a correlation coefficient of $r=0.85$ (see Fig. 5). Taking into

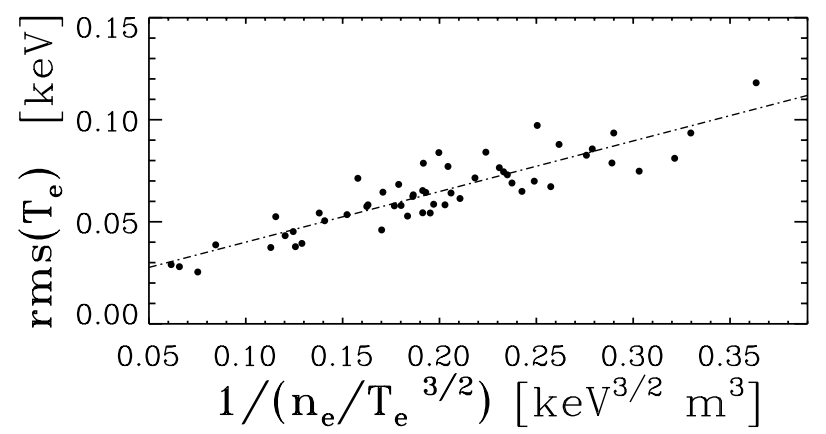

FIG. 5. Global $\operatorname{rms}(T)$ as a function of collisionality for 54 different shots. account that $\operatorname{rms}(T)$ will also increase when the density decreases due to the fact that the measure is less accurate for low density, the dependence purely on the temperature has been studied. The behavior obtained is $\operatorname{rms}(T) \alpha T^{3 / 2}$ with a correlation coefficient of $r=0.90$. These results show that the structures are less pronounced at high collisionality. From the collisionality dependence and the observation that the structures are less pronounced outside the power deposition area, it can be said that the kinetic properties of the plasma could have some influence on the structure's origin [6].

Time resolved electron temperature measurements are obtained in TJ-II using a multichannel heterodyne radiometer [7]. The temporal resolution for the ECE measurements corresponds to $50 \mathrm{kHz}$ video. The spatial resolution along the major radius is of the order of $1 \mathrm{~cm}$ and the vertical spatial resolution, as determined by the focused beam waist, is $\approx 3 \mathrm{~cm}$. A good agreement is obtained between ECE and the smoothed TS temperature profiles, since the available ECE diagnostic resolution does not allow one to detect the large temperature perturbations that appear in the TS profiles. However, significant and fast temperature oscillations, with frequencies of the order of tens of $\mathrm{kHz}$, are always observed in the central ECE channels. The temperature oscillations decrease from the central to the outer channels (see Fig. 6). The $\operatorname{rms}(T(r))$ calculated using the ECE profiles in the same series of 15 reproducible discharges considered above is plotted, and the values are well above the thermal noise of the diagnostic (of about 1.4\%). The fact that the rms of the fluctuations is lower than in the TS system can be explained by the spatial averaging of each ECE channel and the fact that the TS samples over a very short time interval. Large electron temperature fluctuations have been previously observed by a high-resolution ECE radiometer in the tokamak TEXT-U [8]. These phenomena were interpreted in terms of rotating low order modes [9], but these structures appear in TJ-II even when no low order resonances are present.

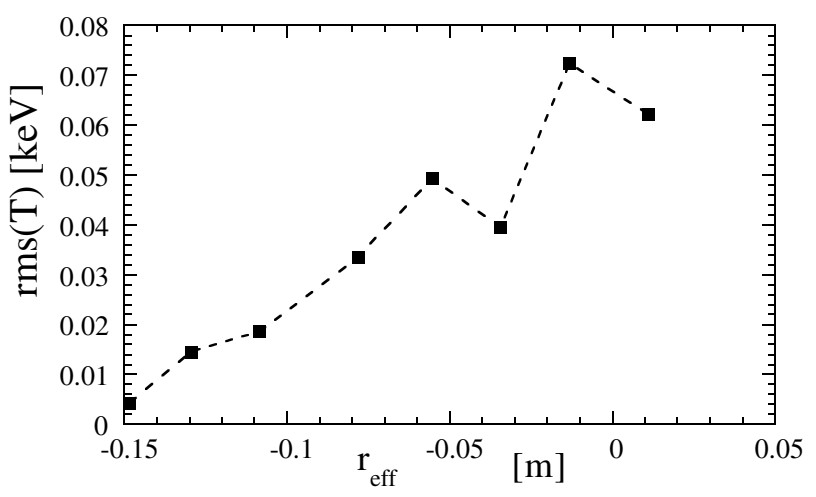

FIG. 6. Calculated $\operatorname{rms}(T(r))$ for the same series as in Fig. 3, but the temperature is now measured by the ECE system. 

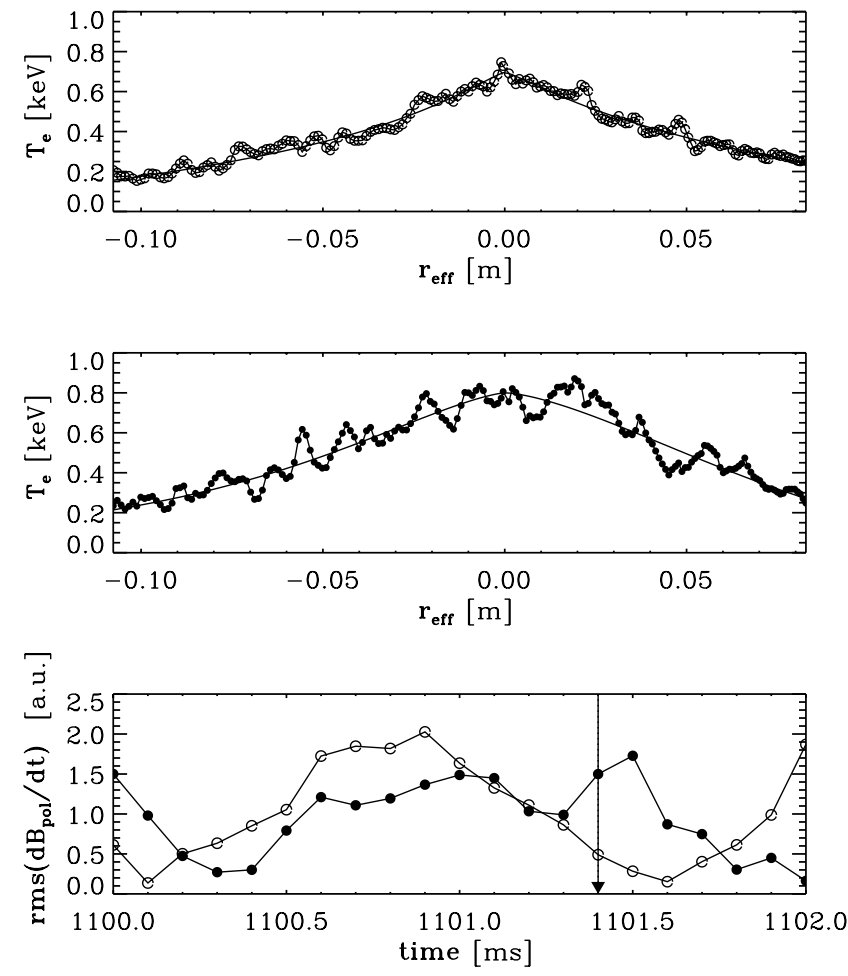

FIG. 7. Comparison of global $\operatorname{rms}(T(r))$ calculated for the low activity shot No. 2151 (top panel, open circles) and high activity discharges shot No. 2157 (middle panel, filled circles), with the fitted functions (drawn lines). The bottom panel shows the time evolution of rms of MHD activity, and the arrow the TS time (open circles for shot No. 2151 and filled circles for shot No. 2157).

Magnetohydrodynamics (MHD) activity measurements, performed with Mirnov coils in TJ-II, present a time response of $100 \mu$ s that is fast enough to account for lasting phenomena in plasmas. It has been observed that the increase of MHD activity coincides systematically with more pronounced structures in the temperature profiles. The rms of MHD activity is plotted in Fig. 7 for two discharges. At TS time the shot No. 2151 presents lower MHD activity than the shot No. 2157. The spectra of these signals show that the change of the activity is due to changes in the amplitude rather than in the frequency. In the case with high activity, the $\operatorname{rms}(T)$ is twice $(59 \mathrm{eV})$ the one with low activity $(29 \mathrm{eV})$ (see Fig. 7). This experimental observation implies that there can be a relationship between magnetic turbulence and profile structures, but further investigation is necessary to find out the origin of the latter.

Both the ECE and MHD activity measurements appear to be sensitive to the structures and show their footprint, as is shown by the coincidence of the trends of fluctuation amplitudes with those measured by TS. The Fourier spectra of MHD activity always present a maximum between 15 and $30 \mathrm{kHz}$ [10], similar to frequencies as detected in the ECE channels. Nevertheless, ECE and Mirnov coil signals are also sensitive to other types of instabilities, such as edge localized mode-like events [10], whose time and radial scales seem to be larger. Therefore, it is necessary to be careful to ensure that the fluctuations of those signals are affected mainly by the structures when only this phenomenon is under study.

Three ideas are considered as contributing to the explanation of possible physical origin of the structures: First, the effects related to magnetic topology of the device, namely, iota profile and resonant surfaces. The fact that some features in the profiles survive to an ensemble average of the series of reproducible discharges would reinforce this theory. However, in order to extract clear conclusions about whether or not the rational surfaces, even the high order ones, have any influence on the profiles, a detailed knowledge of the plasma current profile is needed [10]. Second, the structures can be another manifestation of the wide band plasma turbulence detected by other diagnostics, such as Mirnov coils and the ECE system. Finally, kinetic effects induced by high power density microwaves seem to be able to enhance the structure amplitude. The influence of structures on transport and confinement, if any, must be elucidated with further investigations.

[1] N. J. Lopes Cardozo et al., Phys. Rev. Lett. 73, 256 (1994).

[2] C. Alejaldre et al., Plasma Phys. Controlled Fusion 41, A539 (1999).

[3] C. J. Barth et al., Rev. Sci. Instrum. 70, 763 (1999).

[4] M. N. A. Beurskens et al., Rev. Sci. Instrum. 70, 19992011 (1999).

[5] R. Hertel, in Signal and Noise Properties of ProximityFocused Image Tubes, edited by Gary L. Stradling, SPIE Proceedings Vol. 1155 (SPIE-International Society for Optical Engineering, Bellingham, WA, 1990), pp. 332-343.

[6] V. Krivenski, in Proceedings of the 26th Conference on Controlled Fusion and Plasma Physics, Maastritch, 1999, edited by B. Schweer, G. Van Oost, and E. Vietzke (EPS, Geneva, 1999), Vol. 23J, p. 385.

[7] E. de la Luna et al. (to be published).

[8] G. Cima et al., Plasma Phys. Controlled Fusion 40, 11491158 (1998).

[9] F. Porcelli et al., Phys. Rev. Lett. 82, 1458 (1999).

[10] I. García-Cortés et al. (to be published). 